\title{
El uso de las TIC en la educación universitaria: motivación que incide en su uso y frecuencia
}

\author{
Yohanna Abarca Amador \\ Escuela de Ciencias del Lenguaje \\ Instituto Tecnológico de Costa Rica
}

\begin{abstract}
Resumen
El presente artículo muestra las respuestas a las siguientes preguntas de investigación: ¿Qué motiva a los docentes a utilizar herramientas tecnológicas (TIC) como wikis, foros y blogs? ¿Cuáles herramientas tecnológicas utilizan los docentes universitarios con mayor frecuencia? Para obtener las respuestas se empleó un método mixto de dos etapas aplicado de manera secuencial con triangulación de datos. Los instrumentos para esta triangulación fueron un cuestionario (método cuantitativo) y una entrevista (método cualitativo). Los resultados obtenidos evidenciaron que los docentes utilizan con mayor frecuencia herramientas tecnológicas en sus clases cuando poseen las habilidades tecnológicas necesarias y los conocimientos teóricos para manejar las TIC de forma efectiva. Los resultados de esta investigación también sugieren que los docentes muestran no solo una actitud positiva con respecto al uso de las TIC, sino que son conscientes de la importancia de estas en el desarrollo futuro de los estudiantes como profesionales competentes.
\end{abstract}

Palabras claves: tecnologías de la comunicación y la información (TIC), motivación, docentes, herramientas tecnológicas

\begin{abstract}
This study seeks anwers to the following research questions: What motives teachers to use technological tools such as wikis, forums, and blogs? Which technological tools do teachers use at college level most frequently? To obtain the anwers, a two-stage mixed method was used in a sequential way with data triangulation. The instruments used for this triangulation were a questionnaire (quantitative method) and an interview (qualitative method). The results obtained showed that teachers use technological tools more frequently when they have theoretical knowledge and the necessary technological skills to use information technologies (IT) effectively. The results of this study also suggest that teachers not only show a positive attitude towards the use of IT, but are also aware of their importance in the future development of the students as competent professionals.
\end{abstract}

Key words: information technologies (IT), motivation, teachers, technological tools 


\section{Introducción}

$\mathrm{L}$ as tecnologías de la información y comunicación (TIC) se han convertido en herramientas fundamentales en diversos aspectos de la vida actual. Es difícil concebir el mundo sin computadoras que permitan el acceso a Internet, a las redes sociales y a todos los medios de comunicación virtuales que facilitan el contacto y colaboración entre personas de diferentes nacionalidades, culturas, creencias y contextos sociales.

Por tanto, el surgimiento de nuevas tecnologías y formas de trabajo ha creado la necesidad de que los profesionales, y entre ellos muy especialmente los docentes, desarrollen habilidades y competencias para que puedan utilizar las herramientas tecnológicas de forma efectiva. Al considerar que la educación superior enfrenta el reto de formar profesionales y ciudadanos capaces de afrontar las demandas del ámbito laboral de la sociedad del siglo XXI, Álvarez y Villardón (2006) afirman que uno de los retos de las universidades es formar a personas capaces de construir su propio conocimiento en forma autónoma, por lo cual los docentes actuales deben poseer una serie de características que los hagan desempeñarse satisfactoriamente en la clase y en su vida cotidiana..

Formar a los estudiantes para que se desempeñen en la sociedad actual es una labor difícil que requiere de un cambio radical no solo en la actitud y el papel del estudiante sino también en el rol del docente. Para tal fin, muchos docentes han hecho ingentes esfuerzos por alejarse de la enseñanza tradicional para adoptar un rol más innovador. Estos docentes han tratado de incorporar el uso de herramientas tecnológicas a las que pueden tener acceso, ya sea mediante plataformas educativas en sus instituciones o por medio de Internet. Como resultado de tales esfuerzos, una de las nuevas formas de enseñanza que ha surgido para tratar de incluir el uso de las diversas herramientas tecnológicas es blended learning o aprendizaje combinado. Esta nueva forma de enseñar combina la clase presencial con el uso de herramientas tales como la instrucción interactiva basada en la web, la comunicación vía correo electrónico, los foros de discusión electrónicos, los contenidos para el trabajo autónomo y colaborativo, los exámenes en línea y las audio y videoconferencias.

Mortera (2007) indica que todos estos medios tecnológicos permiten flexibilidad en la discusión de los contenidos educativos. Este autor señala que la interactividad de los medios llega a un mayor número de usuarios o estudiantes que en la instrucción presencial. Ante esta perspectiva, Mortera afirma que la tecnología actual hace posible utilizar una amplia gama de herramientas digitales aun en instituciones que no cuentan con plataformas educativas propias. Además, con estas herramientas tecnológicas los estudiantes no tienen que movilizarse de sus casas para buscar información, hablar con el docente o trabajar en colaboración con un compañero que puede estar al otro lado del planeta. Estos recursos desarrollan la comunicación escrita, el debate de ideas, la exposición de opiniones y una mayor riqueza de contenidos para la enseñanza y el aprendizaje. Sin embargo, para que estos medios se aprovechen y se pongan en práctica son fundamentales su conocimiento y su uso efectivo por parte del docente. 
Para responder a las nuevas demandas tecnológicas, los docentes deben procurar una formación continua basada en la reflexión y el análisis de la práctica educativa. Esto incluye el intercambio de experiencias en el aula y el aprendizaje de estrategias metodológicas entre los docentes mismos, además de la disposición del docente a realizar cambios significativos en sus prácticas educativas, lo cual reviste particular importancia. Muchos docentes continúan enseñando de la manera tradicional sin dar importancia a los cambios que la tecnología ha generado en la educación. A este respecto, Escudero (2008) afirma que estos cambios deben ser asumidos por "instituciones y sujetos que los piensen, los discutan, los asuman reflexivamente y los pongan en práctica”. Si los docentes no deciden utilizar metodologías innovadoras que incluyan el uso de las TIC, los cambios, aunque necesarios y significativos en el aula, no se podrán llevar a cabo.

\section{Manejo de las TIC}

Una de las demandas actuales del currículo universitario es el manejo efectivo de las TIC por parte de los docentes con el fin de fomentar prácticas y habilidades que los estudiantes necesitarán en sus puestos de trabajo. En este sentido, Burgos (2007) indica que "la tecnología aplicada a la educación es un conjunto de teorías y técnicas que permiten ofrecer un proceso mediante el cual se pueden operar herramientas, cambiar, manipular y controlar un ambiente de aprendizaje." (Burgos, 2007, p.261). No obstante, la sola existencia de herramientas tecnológicas no implica mejoras en las metodologías. Es necesario que los docentes se involucren directamente con ellas. Sin embargo, como lo indica Marquès (2007), aunque existe una gran cantidad de nuevas tecnologías disponibles para los docentes, esto no supone el fin de los aprendizajes basados en la memorización o en la simple reproducción de los contenidos. A pesar de esto, el mismo Marquès señala que la disponibilidad de las TIC sí implica que haya cambios importantes como los que se detallan a continuación.

En primer lugar, con la ayuda de Internet los estudiantes tienen la opción de conocer sobre una temática dada desde diversas perspectivas. El docente ya no es el mayor depositario de los conocimientos relevantes de esa temática. En realidad, su papel ahora es el de un asesor o guía, ya que se debe enfocar más en darle a los estudiantes herramientas para la búsqueda inteligente, el análisis crítico y la selección y aplicación de los conocimientos que en su transmisión.

En segundo lugar, el docente tiene menos oportunidades de desarrollar un programa obsoleto. Los estudiantes pueden consultar en Internet lo que se hace en otras universidades y pueden pedir que se les dé una formación similar.

En tercer lugar, los estudiantes pueden realizar sus procesos de aprendizaje de forma más significativa, partiendo de sus conocimientos y experiencias previas, porque tienen a su alcance mucha información para elegir y la posibilidad de solicitar y recibir en cualquier momento el asesoramiento de docentes y compañeros (Marquès 2007). 


\section{Ventajas de usar TIC en la clase}

Burgos (2007) afirma que el uso de nuevas herramientas tecnológicas extiende la aplicación de tecnologías tradicionales como el uso de videos, pizarra o libros de texto. Así, se crean oportunidades para que los estudiantes puedan realizar simulaciones de problemas reales y prácticos. Además, la interacción que se da entre docentes y estudiantes, o entre los mismos estudiantes, fomenta el aprendizaje activo al poder ellos participar en forma autónoma o colaborativa en la elaboración de actividades y proyectos retadores y significativos. Por ejemplo, Muñoz (2008) sostiene que por medio de Internet se pueden conformar redes de aprendizaje a través de una plataforma tecnológica y entonces hacer uso de distintas aplicaciones para facilitar la comunicación, la colaboración y el acceso a recursos digitales. Estas plataformas deben ser amigables para no inhibir el desarrollo de las personas en el proceso de aprendizaje (López, 2010).

Con base en lo anterior, se puede afirmar que las TIC han cambiado la forma de hacer docencia. A este respecto, Marquès (2007) asevera que las TIC facilitan la labor del docente, ya que brindan acceso fácil, rápido y seguro a la información y al procesamiento de datos. Esto ha significado una revolución en la educación, pues en el pasado para que un estudiante pudiera acceder a información fidedigna debía desplazarse a una biblioteca. Marquès de nuevo enfatiza el gran aporte que han dado las TIC con respecto a las posibilidades de comunicación sincrónica y asincrónica. Es decir, a través de medios como el correo electrónico, las redes sociales, los chats, Skype y los foros, tanto docentes como estudiantes pueden contactar a cualquier persona alrededor del mundo en el momento en que así lo deseen.

Como consecuencia, en todas las universidades se está modificando la práctica docente para incluir estas nuevas metodologías. Los docentes, según Poblete (2008), están convencidos de que la inclusión es una forma de ayudar a los estudiantes a obtener herramientas que les serán útiles a lo largo de sus vidas. De esta forma, este cambio metodológico conlleva en la clase cambios en el modo de pensar y actuar tradicionales. Con la ayuda de las TIC, se puede realizar una gran cantidad de actividades para apoyar los contenidos temáticos. A la vez, como se dijo anteriormente, se incrementa la cantidad de recursos y medios de comunicación que los estudiantes tienen para comunicarse en forma digital con el docente y con otros compañeros.

\section{Actividades de clase apoyadas con TIC}

Marquès (2007) recomienda a los docentes universitarios realizar actividades con ayuda de las TIC las cuales incluyan:

1. La elaboración y mantenimiento de una página web de la signatura donde el docente puede tener, por ejemplo, el programa del curso, el calendario de actividades y diversos documentos para los alumnos. 
2. El uso de aparatos y programas informáticos de uso general. Por ejemplo, el entorno Windows, procesador de textos, navegador de Internet y correo electrónico.

3. El conocimiento de las aplicaciones que ofrece el "campus virtual" de la propia universidad en caso de que haya uno.

4. La aplicación de las TIC a la enseñanza como instrumento de innovación didáctica. Algunas actividades específicas en relación con este punto son: la creación de la página web de la asignatura, la organización de tutorías virtuales con los estudiantes, el aprovechamiento de los recursos de Internet para la clase y para las actividades con los estudiantes.

5. El conocimiento y la utilización de las bases de datos y programas informáticos específicos de la materia que se imparte (instrumento profesional).

La realización de todas estas actividades implica para el docente no solo la necesidad de mantenerse una constante actualización de conocimientos sobre el uso eficiente de las TIC. También requiere de muchas horas más de dedicación docente del profesorado. Este es uno de los factores que en ocasiones hace poco atractivo para los docentes el uso de estas herramientas en la clase.

\section{Blended learning: Aplicación de las TIC en la clase}

Uno de los nuevos métodos que se están utilizando para incluir en mayor o menor grado herramientas tecnológicas es blended learning o aprendizaje combinado. Mortera (2007) afirma que el aprendizaje combinado mezcla la instrucción presencial con sistemas propios de la educación a distancia. Algunos de los objetivos de este método son:

1. La variedad pedagógica: Se logra por medio de la combinación de diversas herramientas y actividades en clase o en línea.

2. El acceso al conocimiento: En los cursos tradicionales, el uso de tecnologías es un aporte significativo para que una gran cantidad de estudiantes pueda acceder a conocimientos que antes no estaban a su alcance.

3. La interacción entre docentes y alumnos: La interacción ya no tiene que ser solo sincrónica sino que puede realizarse por otros medios como chats, foros y blogs de forma asincrónica.

4. La reducción de los costos en una educación de calidad: El uso de herramientas tecnológicas provee más oportunidades de interacción, mayor acceso a materiales y conocimiento sin una infraestructura administrativa onerosa, lo cual reduce considerablemente la inversión en educación.

5. La facilidad en el seguimiento: Por medio de Internet, los docentes y estudiantes pueden saber de forma inmediata quién ha participado en una actividad y pueden dar seguimiento a los aportes de cada persona con facilidad. 
El aprendizaje combinado varía dependiendo del grado de participación, tanto de la tecnología que se involucre en el curso, como del componente presencial de este. Mortera (2007, p.144) describe algunos de los ambientes más comunes en el aprendizaje combinado:

1. La instrucción cara a cara o presencial

2. La instrucción interactiva basada en la $W e b$

3. La comunicación vía correo electrónico

4. Los foros de discusión electrónicos

5. El uso de contenidos para aprendizaje autónomo

6. El uso de software para realizar trabajos colaborativos

7. Los salones de clases virtuales

8. Los exámenes en línea

9. El uso de videoconferencias

10. Las audioconferencias

11. Las conferencias vía computadora

Smirnov y Bogun (2011) añaden que estas herramientas promueven el trabajo colaborativo y autónomo, la discusión, el reforzamiento del análisis y el pensamiento científico.

\section{El rol de la motivación en el uso de TIC en la educación}

Marquès (2000) afirma que el uso de herramientas tecnológicas en la educación se ha convertido en un instrumento cada vez más indispensable en las instituciones educativas. Es precisamente en el aula donde cumplen con importantes funciones: fuentes de información, canales de comunicación interpersonal y plataformas del trabajo colaborativo. Además, las herramientas promueven el intercambio de información e ideas por medio de foros, e-mails, blogs y wikis. Por otro lado, las TIC son un medio de expresión para la creación de textos, gráficos, páginas web, presentaciones multimedia y vídeos. Como se puede notar, los recursos interactivos de multimedia que ofrecen estas herramientas informan, entrenan, simulan, guían aprendizajes y motivan a los estudiantes y docentes en un proceso de enseñanza-aprendizaje ágil, efectivo y moderno. Asimismo, una de las mayores ventajas de utilizar TIC, según Marquès, es el interés y la motivación que muestran los estudiantes al tener recursos tecnológicos novedosos a su disposición.

Afines al pensamiento de Marquès (2000), Manassero y Vásquez (2000) se refieren a la motivación en su relación con las TIC. Según ambos autores, la motivación promueve el aprendizaje y las actividades que se realizan en la escuela. Y si la motivación es promovida con el uso de las TIC, sobre todo en los primeros años de instrucción, influye de manera decisiva en la obtención de logros académicos. Por lo tanto, los modernos enfoques cognitivos han convertido la motivación en uno de los constructos centrales en el aula. De hecho, una cantidad considerable de estudios relaciona la motivación con actitudes positivas por 
parte de los estudiantes tales como la curiosidad y la perseverancia. El nivel de motivación de una persona con respecto a una actividad educativa puede influir, por tanto, en el esfuerzo, las emociones positivas, la adaptación psicológica en clase, la calidad del aprendizaje, el interés, la concentración y la satisfacción general con la educación. Por tal razón, para Manassero y Vásquez (2000), la implementación de las TIC en el aula es clave para una actitud empática constante por parte del estudiante hacia el aprendizaje.

La incorporación de las TIC en el aula es, sin embargo, un aspecto complejo de la labor educativa. De hecho, es uno de los mayores desafíos en la práctica docente universitaria, pues constituyen un medio para desarrollar en los estudiantes las capacidades y conocimientos que podrán utilizar como futuros profesionales en sus entornos laborales. A pesar de esto, poco se ha discutido sobre el uso de las TIC por parte de los docentes de este nivel y la motivación por la que se deben emplear estas herramientas tecnológicas en su práctica educativa. A raíz de esta situación, surgió esta investigación realizada con profesores de la Escuela de Ciencias del Lenguaje de la Sede Central del Instituto Tecnológico de Costa Rica en la Provincia de Cartago. La investigación se basa en el trabajo docente de doce profesores del área de comunicación y trece profesores del área de inglés, y se realizó durante el primer y segundo semestres del año 2012. Se utilizaron un cuestionario y una entrevista con el fin de determinar qué TIC utilizan los docentes del Instituto y con qué frecuencia las utilizan en su práctica educativa.

Como parte de la investigación, se consultaron libros, artículos de revistas electrónicas, ponencias de congresos e informes de educación para conformar la revisión bibliográfica. El cuestionario y la entrevista realizados con los docentes fueron elaborados y validados por la autora con el fin de adecuarlos a los objetivos de la investigación. (El cuestionario y la entrevista se incluyen en este artículo en forma de apéndice). El presente estudio se propone buscar las respuestas a las siguientes preguntas:

- ¿Qué motiva a los docentes a utilizar herramientas tecnológicas en su práctica educativa?

- ¿Cuáles herramientas tecnológicas utilizan los docentes con mayor frecuencia?

- ¿Qué resultados observables se obtienen a propósito de la incorporación de las TIC?

\section{Análisis y discusión de resultados}

A continuación se describen los resultados obtenidos del análisis de las entrevistas y los cuestionarios realizados por la investigadora.

\section{Resultados de la entrevista aplicada a docentes}

En la Tabla 1 se describe el análisis de los datos obtenidos de la entrevista realizada a 10 profesores. 
Tabla 1

Análisis de datos de las entrevistas a profesores

(datos recabados por la investigadora)

Número de docentes entrevistados: 10

Categoría 1: Usa herramientas tecnológicas en clase:

Todos los docentes contestaron afirmativamente.

Categoría 2: TIC usadas en los cursos por los docentes:

- videos (7 docentes)

- páginas o sitios de Internet (5 docentes)

- correo electrónico (4 docentes)

- TEC Digital o Plataforma educativa (4 docentes)

- $\quad b l o g s$ (4 docentes)

- $\quad$ audios (2 docentes)

- presentaciones de Power Point (2 docentes)

- foros electrónicos (2 docentes)

- grabadora (2 docentes)

- wikis (2 docentes)

- video beam (2 docentes)

- Google académico (1 docente)

- Facebook (1 docente)

- mapas conceptuales (1 docente)

- $\quad$ chat (1 docente)

Categoría 3: Motivaciones de los docentes para usar las TIC en clase:

- Hacer que las clases sean más interactivas e interesantes (8 docentes)

- La cultura digital de los estudiantes modernos que los hace estar inmersos en la tecnología (8 docentes)

- Las TIC hacen que los estudiantes comprendan más claramente los contenidos (4 docentes)

- Los profesores deben estar actualizados (2 docentes)

- Darle mayor exposición a los estudiantes al idioma inglés (2 docentes)

Categoría 4: Aspectos que influyen en la elección de herramientas tecnológicas para la clase:

- Los contenidos del curso que esté dando (1 docente)

- Que el material sea relevante para la población (1 docente)

- Atender a las necesidades académicas, profesionales y sociales de los estudiantes (1 docente)

- Evaluar de forma diferente (1 docente)

- Los conocimientos que tenga sobre la herramienta (1 docente)

- Las oportunidades que ofrezca la herramienta para el curso (1 docente)

- Mejorar el trabajo (1 docente)

- Las nuevas generaciones, tendencias, habilidades y recursos (1 docente)

- Que los estudiantes realmente aprendan y se motiven (1 docente)

- Que se logre una comunicación más efectiva y más claridad de contenidos (1 docente)

Categoría 5: Ventajas de trabajar con las TIC en el aula:

- Los recursos con que cuentan algunas aulas de la universidad son muy buenos (2 docentes)

- Es algo innovador (2 docentes)

- El acceso a Internet es posible (1 docente)

- El trabajo se facilita mucho con el uso de las TIC (1 docente) 
- Las clases se hacen más interesantes (1 docente)

- En Internet se encuentra gran cantidad de materiales virtuales (1 docente)

- Las competencias que pueden desarrollarse a través de ellas (1 docente)

- El acceso a Internet es fácil (1 docente)

Categoría 6: Opiniones del porqué no se utilizan las TIC en clase:

- Temor a exponerse frente a los estudiantes y evidenciar que no sabe cómo usar la tecnología (5 docentes)

- Tecnofobia: temor al uso de la tecnología (4 docentes)

- No saben cómo utilizar las herramientas (4 docentes)

- Fue la forma como aprendieron (1 docente)

- Es el paradigma de enseñanza del docente (1 docente)

- Es mucho trabajo planear las clases (2 docentes)

En la Categoría 1, la totalidad de los docentes entrevistados afirmó utilizar las TIC en sus clases. En la Categoría 2, se muestran las actividades que los docentes indicaron como las que utilizaban en sus clases ayudados por las TIC. Algunas actividades se mencionaron con mayor frecuencia que otras. Por ejemplo, el uso de videos se mencionó más de la mitad de las veces mientras que el uso de Facebook, mapas conceptuales y chat fueron mencionados únicamente por un profesor. Sin embargo, todos los profesores entrevistados afirmaron utilizar una variedad de recursos tecnológicos. De igual manera, los docentes mencionaron diferentes motivaciones para utilizar las TIC en sus clases (Categoría 3). Un hallazgo importante es que en la mayoría de los casos se mencionó que a raíz de la incorporación de las TIC: "las clases son más interactivas e interesantes."

Un segundo factor que se citó reiterativamente fue que: “... la cultura digital de los estudiantes modernos los hace estar inmersos en la tecnología." El tercer aspecto que se mencionó con más frecuencia fue que las TIC: “...hacen que los estudiantes comprendan más claramente los contenidos." Estos tres aspectos están relacionados y representan un punto de convergencia con Marquès (2007), quien afirma que con la ayuda de las herramientas tecnológicas los estudiantes tienen la opción de conocer sobre una temática desde diversas perspectivas y hacer que sus aprendizajes sean más significativos.

Con respecto a la Categoría 4, los profesores entrevistados mencionaron diversos aspectos a tomar en consideración a la hora de elegir las TIC para sus clases. En la mayoría de los casos, ellos manifestaron que utilizaban las herramientas que fueran relevantes para los cursos en relación con los contenidos por desarrollar o reforzar. Señalaron como requisito que las TIC debían ser a la vez atractivas para los estudiantes. Además, manifestaron un interés por la utilización las TIC como una forma de mantener actualizada su práctica docente.

Con respecto a las ventajas que tienen los docentes al trabajar con las TIC en el aula (Categoría 5), se mencionó que: "Una ventaja son las competencias que pueden lograrse..." Se pueden desarrollar habilidades y conocimientos debido al acceso a una variedad de herramientas y materiales. De igual forma, los profesores mencionaron que el uso de las TIC hace las clases innovadoras e 
interesantes, ya que los recursos tecnológicos proveen a los profesores con una amplia gama de materiales que les ayuda a reforzar los contenidos de su clase.

Finalmente, con respecto a la Categoría 6, la mitad de los docentes entrevistados afirmaron que algunos no utilizan las TIC por temor a exponerse frente a los estudiantes y mostrar que no saben utilizar la tecnología. Esto queda evidenciado en el comentario de una docente quien al consultársele sobre este tema contestó que existen profesores que no utilizan las TIC porque: "Les da miedo que los estudiantes se vayan a burlar de ellos y prefieren hacer lo mismo de siempre."

Las anteriores aseveraciones nos llevan a pensar en la posición de Burgos (2007) sobre las TIC. Para este investigador, la sola existencia de herramientas tecnológicas no implica mejoras en las metodologías. Es necesario que los profesores se involucren directamente con ellas y, por consiguiente, las pongan en práctica en la clase. Por tanto, es indispensable que los docentes reciban una adecuada capacitación en el uso de las TIC para utilizarlas en la clase con toda confianza.

\section{Resultados del cuestionario aplicado a los docentes}

Como respuesta a la pregunta 1 sobre si utilizan herramientas tecnológicas en el aula, el 100\% de los docentes contestó en forma afirmativa. En la Figura 1 se muestran las herramientas que los docentes marcaron como las utilizadas por ellos en sus cursos como respuesta a la pregunta 2. Esta pregunta era de opción múltiple. El porcentaje corresponde al número de docentes que marcó cada herramienta como utilizada en los cursos que imparten en la universidad.

Figura 1

Herramientas tecnológicas utilizadas por los profesores

(Datos recabados por la investigadora)

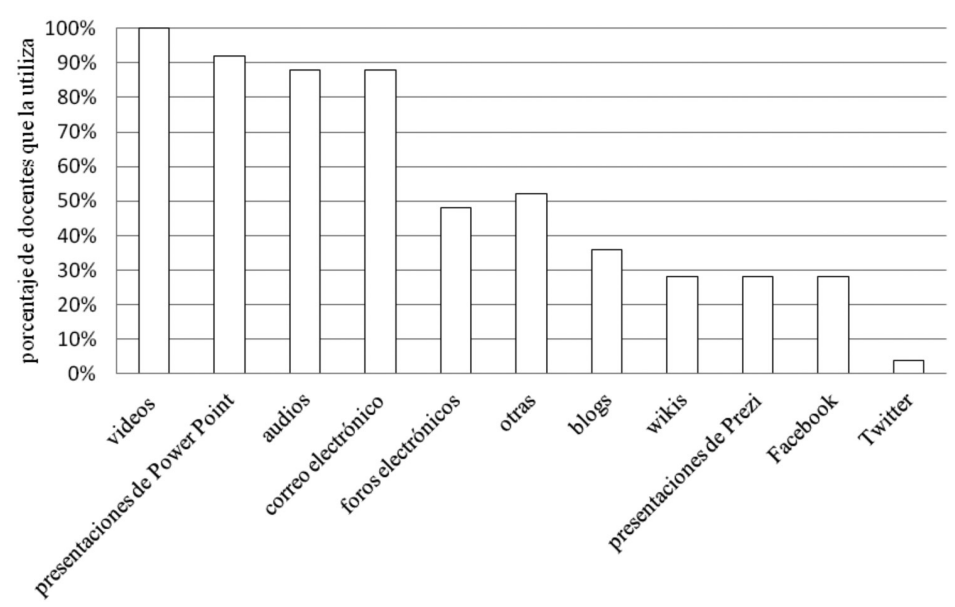


El 100\% de los docentes marcó la herramienta video como utilizada en la clase. La segunda herramienta más marcada fueron las presentaciones de Power Point con un $92 \%$, seguida del uso de audios y correo electrónico con un $88 \%$. La casilla que se refiere a otras herramientas obtuvo un porcentaje de $52 \%$. En otras palabras, poco más de la mitad de los docentes utiliza herramientas distintas de las mencionadas en el cuestionario. De este porcentaje, el $24 \%$ se refiere al uso de páginas o sitios de Internet como You Tube, Google docs, o Googlet. Un 8\% se refiere al uso del TEC Digital, la plataforma digital de la universidad. El 4\% se refiere al uso de pizarras interactivas, otro $4 \%$ se refiere al uso de Podcasts, otro $4 \%$ al uso de softwares, $4 \%$ al uso del celular y, finalmente, otro $4 \%$ al uso de cámaras de video. El uso de foros electrónicos obtuvo un $48 \%$ y los blogs un $36 \%$. Las herramientas menos utilizadas son las wikis, Facebook y las presentaciones con Prezi, con un 28\% respectivamente. Finalmente, el Twitter solo obtuvo un $4 \%$, lo que equivale a que solo un docente de los involucrados en el estudio lo utiliza en clase.

\section{TIC utilizadas con mayor frecuencia por los docentes}

La pregunta número 3 de la encuesta le pedía a los docentes marcar con una equis la frecuencia con que ellos utilizan las distintas herramientas tecnológicas en sus cursos. Esta pregunta tenía como propósito investigar si el uso de las TIC es una práctica frecuente para ayudar a los estudiantes a desarrollar distintos conocimientos y habilidades en sus carreras.

La figura 2 muestra la frecuencia con que los 25 docentes encuestados utilizan distintas herramientas tecnológicas en sus clases universitarias.

Figura 2

Frecuencia con que los profesores utilizan las TIC

(Datos recabados por la investigadora)

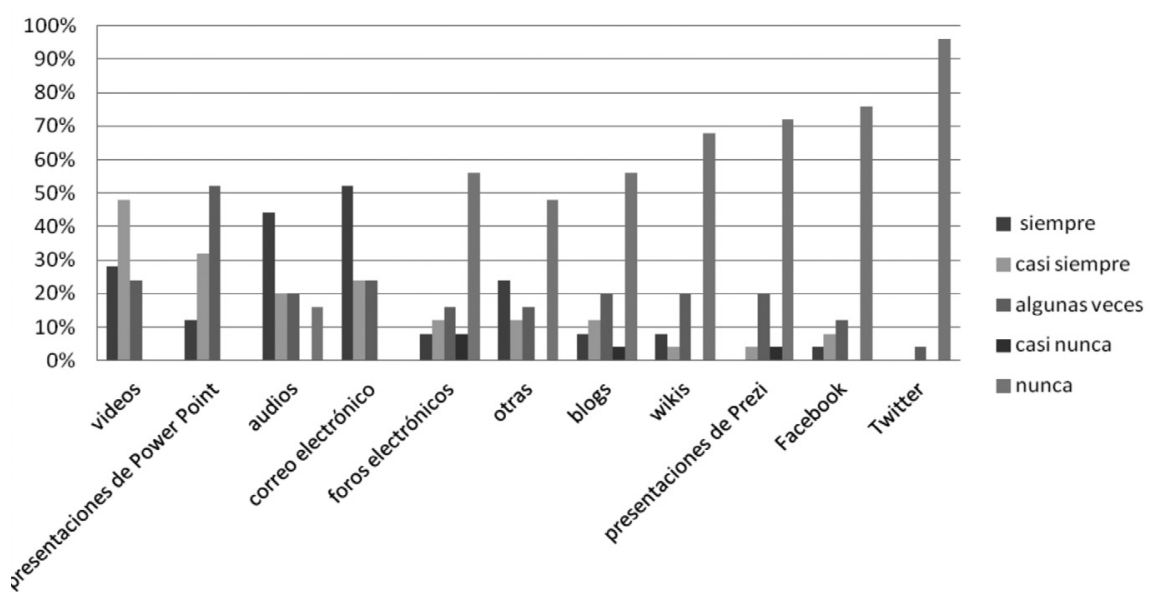


En relación con la frecuencia de uso de las distintas herramientas, el de videos obtuvo un $48 \%$ en la categoría (2) casi siempre, y un $28 \%$ en la categoría (1) siempre. El uso de audios obtuvo un $44 \%$ en la categoría (1) y un $20 \%$ en la categoría (2). Las presentaciones de Power Point obtuvieron un 52\% en la categoría (3) algunas veces, y un 32\% en la categoría (2). El correo electrónico obtuvo un $54 \%$ en la categoría (1) y un $24 \%$ en las categorías (2) y (3).

Un hallazgo interesante es que si bien los docentes marcaron casi todas las herramientas como utilizadas en clase, la frecuencia con que utilizan muchas de ellas no es tan alta. Varias herramientas obtuvieron el porcentaje más alto en la categoría (5) nunca. Por ejemplo, Prezi obtuvo un 72\% en esta categoría; los foros electrónicos un 56\%; las wikis un 68\%: los blogs un 56\%; Facebook un 76\% y Twitter un $96 \%$.

Estos resultados reafirman en un ciento por ciento los resultados obtenidos en la entrevista. No existen diferencias significativas con respecto a las herramientas que utilizan los docentes y a la frecuencia con que las utilizan en la clase.

\section{Motivación para utilizar las TIC en la clase}

Sobre la pregunta abierta número 5: ¿Qué motiva a los docentes a utilizar herramientas tecnológicas en sus clases?, las respuestas que más se repitieron fueron las siguientes:

1. Clases más interesantes y dinámicas (18)

2. Utilización de una amplia gama de recursos innovadores (9)

3. Actitud positiva de los estudiantes hacia las TIC (8)

4. Buen manejo de la tecnología por parte de los estudiantes (5)

5. Contenidos más fáciles de entender (4)

La motivación principal de los docentes para utilizar las TIC, de acuerdo con las respuestas suministradas en el cuestionario, la constituye la posibilidad de impartir clases más interesantes y dinámicas. La actitud positiva de los estudiantes hacia las TIC también se mencionó varias veces como una razón para utilizarlas. Al respecto, en un estudio sobre las actitudes de los estudiantes universitarios con respecto a las TIC, Tingoy y Serkan (2011) encontraron que la mayoría de los estudiantes estuvieron de acuerdo en que las tecnologías de la comunicación y la información son una parte indispensable de la educación. Esta aseveración apoya la idea de que los estudiantes saben de la importancia del uso de las TIC en la actualidad y, en su mayoría, muestran una actitud positiva hacia ellas.

Otra de las razones que se externó en el cuestionario, como una motivación para usar las TIC, fue la amplia gama de recursos innovadores que estas ofrecen para su utilización en clase. Asimismo, 4 docentes consideraron que las TIC: "facilitan el aprender la materia, y "son más variadas las prácticas para los estudiantes”. Así pues, al comparar la motivación por parte de los docentes, 
expresadas en las entrevistas y los cuestionarios con respecto al uso de las TIC en clase, se evidencia que la motivación más importante mencionada en ambos instrumentos es "clases más interesantes y dinámicas". Como lo indica Marquès (2007), el uso de las TIC motiva a los estudiantes a aplicar los recursos tecnológicos como parte de su desempeño en clase, a la vez que dedican más tiempo al trabajo y al aprendizaje. Es importante agregar aquí que los resultados obtenidos en este estudio indican que la motivación docente está muy relacionada con la posibilidad de ofrecer cursos con más recursos de aprendizaje, por lo tanto, más interesantes y dinámicos.

Todos los docentes que participaron en la investigación mostraron una actitud positiva con respecto al uso de las TIC en la clase. Esto justicia que el 100\% de los docentes incluidos en el estudio utilizan diversas herramientas tecnológicas y afirman que su uso es indispensable en la era tecnológica actual. Asimismo, las herramientas tecnológicas que obtuvieron una mayor frecuencia de uso son las que no requieren de mucha complejidad teórica o tecnológica. En otras palabras, los docentes utilizan más frecuentemente las TIC que estén más acordes con sus conocimientos y habilidades. Por ejemplo, los recursos de audio y video, las presentaciones en Power Point y los correos electrónicos son los recursos más usados de acuerdo con los resultados. Sin embargo, también hay docentes en el estudio con más capacitación tecnológica quienes están utilizando herramientas aún más innovadoras como blogs, wikis y foros.

Con respecto a los resultados anteriores, se debe también recordar que, como lo indican Haydn y Barton (2007), los docentes no tienen que saber cómo usar todas las herramientas tecnológicas. Más importante es su interés por buscar herramientas tecnológicas que les sean de utilidad como apoyo para sus clases y, a partir de ahí, empezar a hacer los cambios pertinentes en su práctica docente. Un pequeño cambio basado en las TIC puede empezar a marcar una diferencia en la práctica educativa de los docentes y en la forma en que los estudiantes pueden desarrollar sus conocimientos y habilidades. Como lo indican Paolini, Rinaudo y Donolo (2004), una nueva gama de posibilidades se abre para la docencia universitaria con el uso de nuevas metodologías y con la incorporación de las TIC. Además, es necesario reiterar que, como ha quedado demostrado a través de este estudio, las TIC son un medio eficaz para motivar a los estudiantes en sus procesos de enseñanza y aprendizaje.

\section{Conclusiones}

Se concluyó de las entrevistas y el cuestionario que se utiliza un continuo de TIC en las aulas universitarias. Este continuo incluye desde el uso de presentaciones de Power Point, audios y videos hasta el uso de podcasts, sitios web, foros y Facebook. El 100\% de los docentes utilizan distintas herramientas tecnológicas del continuo en sus cursos y afirman que el uso de TIC es indispensable en la sociedad tecnológica en que estamos inmersos. Sin embargo, el tipo de TIC y su frecuencia de uso va a depender de los conocimientos que posea el docente y las 
necesidades de contenidos que se necesite enseñar en sus cursos. Cabe mencionar que las herramientas tecnológicas que obtuvieron una mayor frecuencia de uso siguen siendo en su mayoría TIC más tradicionales como, el uso de videos, audios, presentaciones de Power Point y correo electrónico.

De los resultados obtenidos se puede inferir que esto ocurre debido a que las TIC no requieren de mucha complejidad teórica o tecnológica para su manejo. Sin embargo, también se evidencia que existe un número importante de docentes que están utilizando TIC más novedosas y complejas como son los blogs, wikis y foros. Estas herramientas ofrecen otro tipo de interacción y posibilidades tanto al docente como a los estudiantes universitarios. A partir de estos hallazgos, el presente estudio sugiere que se están llevando a cabo cambios en la labor docente universitaria en forma paulatina con la incorporación de TIC en las aulas. Se vislumbra un cambio positivo en la forma en que los estudiantes desarrollan sus procesos educativos en la universidad.

Con respecto a qué motiva a los docentes para utilizar TIC en clase, hay tres razones principales expresadas por ellos en el estudio: (1) las herramientas crean espacios más interesantes y dinámicos, (2) ofrecen recursos innovadores y (3) mejoran la actitud de los estudiantes hacia los contenidos del curso. La evidencia obtenida a partir de los cuestionarios y las entrevistas apunta a que la motivación de los estudiantes es el motor que hace que los docentes utilicen las TIC. Sin embargo, la motivación hacia el uso de las TIC no es exclusiva de los estudiantes. De hecho, los docentes en el estudio han mostrado su motivación al respecto. Este factor conlleva el deseo por parte de los docentes de actualizarse e innovar las clases. Así, el uso de las TIC es un recurso muy valioso para promover el desarrollo integral de los estudiantes universitarios para poder ser competentes y efectivos en una sociedad en la cual el conocimiento y la tecnología van de la mano en una misma ruta.

\section{Bibliografía}

Álvarez,Y. y Villardón, L. (2006). Cuadernos Monográficos del ICE: Planificar desde competencias para promover el aprendizaje. Bilbao, España: Universidad de Deusto.

Burgos, J. (2007). El reto de la radio interactiva y la tutoría virtual. En A. Lozano y B. Vladimir, Tecnología Educativa en un Modelo de Educación a Distancia Centrado en la Persona (pp. 241-276). México: Limusa.

Escudero, J. (2008). Las competencias profesionales y la formación universitaria: posibilidades y riesgos. Revista de Docencia Universitaria, II, 3-20. Recuperado de http://www.redu.m.es/Red_U/m2.

Haydn,T. y Barton, R. (2007). 'First do no harm': developing teachers' ability to use ICT in subject teaching: some lessons from the UK. British Journal of Educational Technology, 38(2), 365-368. doi:10.1111/j.1467-8535.2006.00639. http://onlinelibrary.wiley.com/doi/10.1111/j.14678535.2006.00639.x/abstract ?userIsAuthenticated=false\&deniedAccessCustomisedMessage= 
López Falconi, J. E. (2010). Comunidades de prácticas de valor para el aprendizaje organizacional. En Burgos Aguilar, V. y A. Lozano Rodríguez (Comp.). Tecnología educativa y redes de aprendizaje de colaboración. México: Trillas.

Manassero y Vásquez (2000). Análisis empírico de dos escalas de motivación escolar. REME. Recuperado de http://reme.uji.es/articulos/amanam5171812100/ texto.html

Marquès, P. (2007). Impacto de las TIC en la enseñanza universitaria. Facultad de Educación, $U A B$. 1-15. Recuperado de http://dewey.uab.es/pmarques/ticuniv.htm

Mortera, G. (2007). El aprendizaje híbrido o combinado (Blended Learning): Acompañamiento tecnológico en las aulas del siglo XXI. En A. Lozano y J. Burgos (eds.), Tecnología Educativa en un Modelo de Educación a Distancia Centrado en la Persona (págs. 125-156). México: Limusa.

Muñoz Sheridan, A. (2008). Factores implicados en la conformación de redes escolares con el soporte de un portal educativo: Un enfoque de comunidades de práctica docente. En J. M. Fernández-Cárdenas y C. Carrión-Carranza, Escenarios virtuales y comunidades de práctica. La participación docente en la Red de Escuelas Asociadas a la UNESCO (págs. 95-115). Monterrey: Comité Regional Norte de Cooperación con la UNESCO.

Paolini, P., Rinaudo, M. y Donolo, D. (2004). Estudio exploratorio acerca de tareas académicas en la universidad. R.E.M.E. Revista Electrónica de Motivación y Emoción, 16 (IV). Recuperado de http://reme.uji.es/llista/llista.htm

Poblete, M. (2008, setiembre). El aprendizaje basado en competencias: claves docentes. Trabajo presentado en IV Congreso Internacional: La Renovación de Metodologías Docentes Centradas en el Nuevo Proceso de Aprendizaje del Alumno, Valladolid, España.

Smirnov, E. y Bogun, V. (2011) Science Learning with Information Technologies as a Tool for "Scientific Thinking" in Engineering Education. US China Education Review, 4, 439-463.

Tingoy,O. y Serkan, S. (2011). Informatics Education At Different Disciplines At University Level Case Study: A Survey of Students' Attitude Towards Informatics Technologies. The Turkish Online Journal of Educational Technology, 10, 221-229. 
